\title{
Laser hazards and safety in dental practice: A Review
}

\author{
Meenakshi Boddun ${ }^{1 *}$ and Vijayta Sharva ${ }^{2}$ \\ ${ }^{1}$ Department of Periodontology, People's Dental Academy, Bhopal, India \\ ${ }^{2}$ Department of Public health Dentistry, People's Dental Academy, Bhopal, India
}

\begin{abstract}
The intendment of this review is to give the readers, an insight about the practical guidelines to overcome the possible hazards which can be managed adequately with the proper knowledge of handling the laser device. The article describes about the interaction of laser with the biological tissues, hazards that may commence during the use of laser device, as well as the principle safety rules and regulations.
\end{abstract}

\section{Introduction}

In the past years there has been a large-scale development of the mechanical cutting devices used in dentistry. Despite the considerable progress, dental patients are still apprehensive regarding the noise and vibration produced by the mechanical action of the devices used in dentistry. Starting from the 20th century until now, there has been an unceasing improvement in the development of laser-based dental devices. Once contemplated as a complicated technology with limited uses in dentistry, there is a growing understanding of the utility of lasers in modern dental practice, where they can be used as an adjuvant or substitute to traditional long-established procedures.

Commonly, lasers are classified according to the physical structure (example; solid, gas, liquid state, or semiconductor diode), according to the medium used (example; Erbium: Yttrium Aluminum Garnet (Er: YAG), and the degree of hazard to tissues following inadvertent exposure to lasers. Lasers were accessible commercially for use in dental practice in Australia since 1990, and now the present-day laser units have improved immensely both in terms of technical performance and handling [1,2].

Lasers have been approved by FDA for dental procedures like caries removal, gingivectomy, gingivoplasty, biopsy, coagulation, frenectomies, resection of gingival enlargements, treatment of oral pathologies and polymerization of dental resin materials. Other potential applications include scaling root planning, caries removal, root canal obturation, and dentinal hypersensitivity treatment $[1,3,4]$.

The word laser has a fascinating influence on society and is anticipated to be modern and high tech. However, like all other mechanical procedures, use of laser units requires training and proper handling of the laser armamentarium, overlooking which can be hazardous to both the dentist, dental assistants and the patients.

An adverse event is defined as a serious and undesirable patient experience that results from a medical instrument or product marketed in accordance. Such events include death, life-threatening injury, disability, hospitalization, and congenital anomaly possibly caused by the interaction of the instrument or product during pregnancy, resulting in a birth defect. Specific device problems such as defects, safety, or performance also are reportable.
Dental professionals while using lasers may be in similar inadvertent situation, which can be avoided if proper information of the device and the associated hazards is known by the professional. Laser hazards and safety measures are discussed in detail.

\section{Laser hazards}

Lasers are classified into four broad areas depending on the potential for causing biological damage. When you see a laser, it should be labeled with one of these four class designation [5].

- Class I - These lasers cannot emit laser radiation at known hazard levels.

- Class I.A. - This is a special designation that applies only to lasers that are "not intended for viewing," such as a supermarket laser scanner. The upper power limit of Class I.A. is $4.0 \mathrm{~mW}$.

- Class II - These are low-power visible lasers that emit above Class I levels but at a radiant power not above $1 \mathrm{~mW}$. The concept is that the human aversion reaction to bright light will protect a person.

- Class IIIA - These are intermediate power lasers (cw: 1-5mW), which are hazardous only for intra beam viewing. Most pen-like pointing lasers are in this class.

- Class IIIB - These are moderate power lasers.

- Class IV - These are high power lasers (cw: $500 \mathrm{~mW}$, pulsed: 10 $\mathrm{J} / \mathrm{cm}^{2}$ or the diffuse reflection limit), which are hazardous to view under any condition (directly or diffusely scattered), and are potentials fire hazard and a skin hazard. Significant controls are required of Class IV laser facilities

According to the Centre for Devices and Radiological Health (CDRH) and American National Standards Institute (ANSI) system of classification, Class IV lasers are defined as those devices that pose a

*Correspondence to: Meenakshi Boddun, MDS (Reader), Department of Periodontology, People's Dental Academy, Bhopal, India, E-mail: boddunmeenakshi@gmail.com

Received: December 20, 2019; Accepted: January 07, 2020; Published: January 10,2020 
biologic hazard from either direct or diffuse reflection. Generally, any laser capable of emitting power greater than $500 \mathrm{~mW}$ continuous wave outputs belongs in this class [6-9].

\section{Types of hazards} follows:

The types of hazards that may be encountered may be grouped as

\section{i. Ocular hazards}

Potential injury to the eye can occur either by direct emission or by reflection from a specular (mirror like) surface. Retinal or corneal burn can occur as primarily ocular injury. Retinal injury is possible with emission in the visible light of $400-780 \mathrm{~nm}$ and near infrared of 780-1400nm wavelengths. Direct and specular reflections of relatively low intensity may cause damage because of the focusing response of the lens and cornea. Irreversible retinal burns resulting in permanent blindness can occur by conversion of incident radiation to heat energy within a fraction of a second $[5,10,11]$

\section{ii. Tissue damage}

Ultraviolet lasers (<400 nm wavelength) are not applicable for use in dentistry, as the ionizing risk of these wavelengths is not applicable in a general practice setting. Nonetheless, all other laser wavelengths can cause skin damage (erythema, tissue ablation, tissue irritation and burns) owing to interaction with target chromophores

\section{iii. Respiratory hazard}

The laser plume, which is the smoke or vapor transmitted from the site of surgery during exposure to laser wavelength, is a peculiar concern. The plume should be regarded as hazardous both in terms of particulate matter and infectivity.

Airborne contaminants may be controlled by ventilation, evacuation, or other methods of respiratory protection. Adequate suction in the surgical field must be maintained at all times, especially when treating pathologic conditions that are suspected of being viral in origin to limit the possibility of spreading that virus via the laser plume. The evacuation system should be able to remove particles as small as $0.3 \mu \mathrm{m}$ with at least $80 \%$ efficiency. Surgical staff should wear masks that remove particles as small as $0.3 \mu$. Conventional surgical masks are not adequate, eye wears or face shields and caps and gowns that protect against spatter and laser light should be worn for the protection of operating room personnel $[5,10]$.

\section{iv. Fire, explosion and electric shock}

There are risks associated with the possible heat component arising from the moveable power-driven components of a laser, electric shock and mains supplies (pressurized air, water). Fire risks, via ignition of tubing, some gases or chemicals (e.g. alcohol-based disinfectants) should be identified and avoided $[5,6]$.

\section{Laser safety}

Safety is integral part of providing any dental treatment with a laser instrument. It covers regulations, hazard recognition, device safety, environment, surgical team and target tissue of the patient [5].

The safety measures can be divided into General and Specific safety measures.

\section{General safety measures include:}

\section{i. Environment}

Laser light properties include the concept of beam collimation, with minimal divergence with distance. In practice, laser beams are directed through handpiece systems, which may use either a lens system or focusing or a simple optic fiber. In both cases, some divergence will take place, which results in a distance beyond which the danger posed by the beam is reduced to a secure level.

For lasers, this is called as the nominal hazard distance which is up to few meters. This concept defines a controlled area within which all personnel must be protected. The controlled area must be marked with warning signs that stipulate the risk, surfaces should be non-reflective, doors and windows should be either supervised or operated by remote interlocks during laser procedures [10,11].

Other safe operating methods include the protection of non-target tissue during laser surgery. Reflective instruments and those with mirrored surfaces should be avoided since they can cause damage to non-target tissue.

Shielding may be used as an effective method to avoid inadvertent contact of the beam with tooth enamel or root surfaces. A no. 7 wax spatula or the small end of a Prichard periosteal elevator can be inserted into the gingival sulcus to serve as an effective shield when lasing soft tissue near teeth. Appropriate power settings and time frames are essential to reduce the risks of undesirable laser effects on teeth and supporting structures. Proper knowledge of the specific laser in use and its tissue effects is essential for the operator. Most laser accidents and injuries can be prevented if appropriate control measures are recognized and implemented $[5,10]$.

\section{ii. Safety officers}

The safety officer is appointed to ensure that all safety aspects of laser use are identified and enforced. Ideally, this could be a suitably trained and qualified dental surgery assistant.

Duties of the laser safety officer (LSO) include the following: [10,12]

- Confirm classification of the laser.

- Read manufacturers' instructions concerning installation, use and maintenance of the laser equipment.

- Oversee controlled area and limit access.

- Post appropriate warning signs.

- Recommend appropriate personal protective equipment, such as eye wear and protective clothing.

- Assume overall control for laser use and interrupt treatment if any safety measure is infringed.

- Laser equipment should be properly assembled for use.

- Maintain a log of all laser procedures carried out, relative to each patient, the procedure and laser operating parameters.

- Maintain an adverse effects reporting system-to record any accidents during laser use.

- Oversee maintenance protocols for laser equipment.

- Train workers in the safe use of lasers. 


\section{iii. Local rules}

The local rules should be read and signed by all practice personnel involved in the delivery of laser treatment and updated regularly. It should include:

- Name and address of the practice.

- Each laser used, identified by manufacturer, wavelength, emission mode, power output, delivery system and serial number

- Personnel permitted to use the laser, training and education of personnel involved in the use of lasers.

- Designation of the authority and responsibility for the evaluation and control of laser hazards to a laser safety officer.

- A written laser safety policy, to include Establishment of a quality assurance programme, including regular inspection and maintenance of the laser equipment.

- Management of incidents and accidents, including reporting, investigation, analysis and remedial action.

\section{iv. Training}

All staff members should receive training in the safety aspects of laser use in dentistry, as with other specialties.

\section{Specific safety measures include $[5,10]$}

\section{i. Laser safety features}

All lasers have built-in safety features that control the correct emission of laser energy including:

- Emission port shutters to prevent laser emission until the correct delivery system is attached.

- Covered foot-switches, to prevent accidental operation.

- Control panel to ensure correct emission parameters.

- Audible or visual signs of laser emission.

- Emergency 'stop' button.

- Locked unit panels to prevent unauthorized access to internal machinery.

- Key or password protection.

- Remote interlocks.

\section{ii. Eye protection}

Laser protective eye wear is intended to provide a level of protection that may occur from brief accidental exposures and should not be used to stare directly into the beam. Several varieties are available. Laser protective eye wear should also be clearly labeled or coded to indicate the wavelength and optical density attenuated and should be inspected regularly for degradation of the filter by cracks and pitting. All protection glasses or goggles should be marked with the wavelength for which protection is given, together with a value of optical density (OD). The OD refers to the ability of a protective material to reduce laser energy of a specific wavelength to a safe level below the maximum permitted exposure limit for the eye. The OD value should be at 5.0 or above for adequate protection $[10,12]$

\section{iii. Access}

Maximum numbers of dental surgeries are performed in rooms with barriers walls and one or two entrance doors. Considering such dental operating areas that are multi-chaired and in open environment, would need to direct the requirements of limited access during laser treatment i e; only the clinician, assistant and patient should be allowed within the controlled area.

\section{iv. Test-firing}

Before any laser procedure the clinician should test fire the laser. This affirms that the laser has been assembled correctly and emission of laser occurs through the delivery system. Protective eyewear should be worn and all other safety measures should be done. The laser is directed towards a suitable absorbent material, [e.g. water for long wavelengths and dark colored paper for short wavelengths, longer wavelengths (erbium ysgg, erbium: YAG and co2) can be tested in water, whereas shorter wavelengths that are absorbed by pigments can be test-fired on articulating paper.] and operated at the lowest power setting for the particular laser being used. Following this, the laser is deactivated and the patient is admitted.

\section{Sterilization and infection control}

Steam sterilization is the standard of care. The small flexible optic fibers, handpieces, or tips must be steam sterilized in separate sterilization pouches after each use. They should be kept in the sterilization pouch until ready for use. It is essential that when using fiber-optically delivered lasers, the port (connecting) end remains clean and oil-free. Therefore, never run the fiber in a sterilizer cycle alongside a high-speed turbine with lubricant. If an instrument was used to cleave or recleave a fiber during or after a procedure, then it also must be steam sterilized.

The protective housing around the laser, including the control panel and articulating arm (if applicable) should receive the spray disinfectant/ wipe/ spray disinfectant decontamination method, as do the dental cart and counter tops. Some delivery system components such as the large-diameter erbium fiber-optic cable are not designed for steam sterilization and must be disinfected in this way [5].

\section{Conclusion}

There is ample literature stating the advantages of lasers when used in adjunct and as a single therapy for certain procedures, showing beneficial results like, much less bleeding and less postoperative pain, when compared with conventional procedures utilizing cutting instruments. Beside the affirmative aspect, lasers may also cause damage to the patient as well as the clinician. This article reviews about the paramount safety measures which are to be taken into serious consideration when lasers are used in dental setup. Therefore, when faced with such an unintended event, the clinician should be able to manage the patient adequately. These safety measures ensure adequate emergency care and a safe environment for the practitioner, the patient and the dental assistants.

\section{References}

1. Ishikawa I, Aoki A, Takasaki AA, Mizutani K, Sasaki KM, et al. (2009) Application of lasers in periodontics: true innovation or myth? Periodontol 2000 50: 90-126. [Crossref]

2. Sulewski JG (2011) Einstein's splendid light: Origins and dental applications. In Convissor R A, Principles and practice of laser dentistry. ( $1^{\text {St }}$ Edn), Elsevier pp-1-11.

3. Wigdor H, Abt E, Ashrafi S, Walsh JT Jr (1993) The effect of lasers on dental hard tissues. J Am Dent Assoc 124: 65-70. [Crossref]

4. U.S. Food and Drug Administration (2003) Center for Devices and Radiological Health.

5. Piccione PJ (2004) Dental laser safety. Dent Clin North Am 48: 795-807.

6. American National Standard for the Safe Use of Lasers in Health Care Facilities. ANS (1996) Orlando (FL): Laser Institute of America. 
7. American National Standard for Safe Use of Lasers. ANSI Z136.1-2000. Orlando (FL): Laser Institute of America.

8. United States Food and Drug Administration (2003) Center for Devices and Radiological Health. Good manufacturing practices and quality system regulation.

9. United States Food and Drug Administration (2003) Center for Devices and Radiological Health. CFR 8: 10-11.
10. Beer F, Strabl M, Wernisch J, Laser safety (1995) In: Moritz A, editor, Oral laser application. 2nd Ed. New York: Raven Press 465-478.

11. Morrison A, Conrod S (2009) Dental burs and endodontic files: are routine sterilization procedures effective? J Can Dent Assoc 75: 39. [Crossref]

12. Sliney DH (1995) Laser safety, Lasers Surg Med 16: 215-25.

Copyright: $\odot 2020$ Boddun M. This is an open-access article distributed under the terms of the Creative Commons Attribution License, which permits unrestricted use, distribution, and reproduction in any medium, provided the original author and source are credited. 